\title{
Identification of potential specific biomarkers and key signaling pathways between osteogenic and adipogenic differentiation of hBMSCs for osteoporosis therapy
}

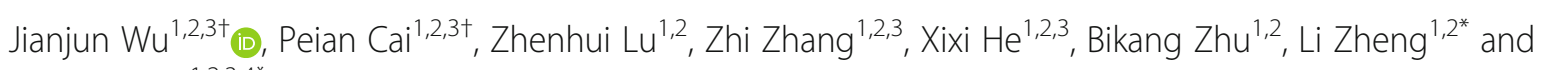
Jinmin Zhao ${ }^{1,2,3,4^{*}}$

\begin{abstract}
Background: The differentiation of bone mesenchymal stem cells (BMSCs) into adipogenesis (AD) rather than osteogenesis (OS) is an important pathological feature of osteoporosis. Illuminating the detailed mechanisms of the differentiation of BMSCs into OS and AD would contribute to the interpretation of osteoporosis pathology.

Methods: To identify the regulated mechanism in lineage commitment of the BMSCs into OS and AD in the early stages, the gene expression profiles with temporal series were downloaded to reveal the distinct fates when BMSCs adopt a committed lineage. For both OS and AD lineages, the profiles of days 2-4 were compared with day 0 to screen the differentially expressed genes (DEGs), respectively. Next, the functional enrichment analysis was utilized to find out the biological function, and protein-protein interaction network to predict the central genes. Finally, experiments were performed to verify our finding.

Results: FoxO signaling pathway with central genes like FoxO3, IL6, and CAT is the crucial mechanism of OS, while Rap1 signaling pathway of VEGFA and FGF2 enrichment is more significant for AD. Besides, PI3K-Akt signaling pathway might serve as the latent mechanism about the initiation of differentiation of BMSCs into multiple lineages.
\end{abstract}

Conclusion: Above hub genes and early-responder signaling pathways control osteogenic and adipogenic fates of BMSCs, which maybe mechanistic models clarifying the changes of bone metabolism in the clinical progress of osteoporosis. The findings provide a crucial reference for the prevention and therapy of osteoporosis.

Keywords: Osteoporosis, Osteogenesis, Adipogenesis, Bone marrow mesenchymal stem cells, Microarray analyses

\footnotetext{
* Correspondence: zhengli224@163.com; zhaojinmin@126.com

†Jianjun Wu and Peian Cai contributed equally to this work.

'Guangxi Engineering Center in Biomedical Material for Tissue and Organ

Regeneration, The First Affiliated Hospital of Guangxi Medical University,

Nanning 530021, China

Full list of author information is available at the end of the article
}

(c) The Author(s). 2020 Open Access This article is licensed under a Creative Commons Attribution 4.0 International License, which permits use, sharing, adaptation, distribution and reproduction in any medium or format, as long as you give appropriate credit to the original author(s) and the source, provide a link to the Creative Commons licence, and indicate if changes were made. The images or other third party material in this article are included in the article's Creative Commons licence, unless indicated otherwise in a credit line to the material. If material is not included in the article's Creative Commons licence and your intended use is not permitted by statutory regulation or exceeds the permitted use, you will need to obtain permission directly from the copyright holder. To view a copy of this licence, visit http://creativecommons.org/licenses/by/4.0/ The Creative Commons Public Domain Dedication waiver (http://creativecommons.org/publicdomain/zero/1.0/) applies to the data made available in this article, unless otherwise stated in a credit line to the data. 


\section{Introduction}

Osteoporosis, characterized by an increase of bone fragility and susceptibility to fracture owning to low bone mass and degeneration of bone microstructure, is a major social problem in the elderly population [1]. Currently, most therapeutic strategies for osteoporosis were administrated by anti-absorption drugs including bisphosphonates, calcitonin and estrogen [2-5]. Although these drugs have a certain effect on delaying the progress of osteoporosis, the serious side effects caused by a long-term use are concerns of clinical application [6-8]. Thus, it is still an urgent need to discover a safer and more effective therapeutic strategy.

Growing evidences indicated that age-related microenvironmental changes including metabolic changes, immune system diseases, and hormonal disorders, reduced the capacity of osteogenic differentiation and strengthened the ability in adipogenic differentiation of BMSCs, which were the paramount motivators in the initiation and progress of osteoporosis [9-12]. Therefore, a regenerative therapy has aroused much attention, which restores the balance of the normal intraosseous microenvironment of osteoporosis by artificially promoting $\mathrm{OS}$ and inhibiting AD from BMSCs. However, the molecular mechanisms of lineage commitment determinants during BMSCs differentiate into $\mathrm{OS}$ and $\mathrm{AD}$ are still unclear.

Adipogenesis and osteogenesis exist a competitive relation during the differentiation of BMSCs, and the signaling pathways between the adipo-osteogenesis could be altered to favor osteoblast for preventing osteoporosis. Adipo-osteogenic differentiation is a process regulated by multiple factors and signals. It has reported that the activation of glucagon-likepeptide1 (GLP-1) not only promotes osteogenic differentiation of BMSCs, but also inhibits adipogenic differentiation which is beneficial for osteoporosis [13]. Other osteogenic factors such as myocardin-related transcription factor A (MRTFA), $\gamma$ glutamyl carboxylase (GGCX), and transducin-like enhancer of split 3 (TLE3) inhibited adipogenic differentiation resulting in amelioration of bone formation [1416]. Although previous studies have revealed possible molecular mechanisms involved in BMSCs to differentiate into $\mathrm{OS}$ and $\mathrm{AD}$, their results are limited to a single lineage and/or a differentiation in late-stages, and the mechanism in the differentiation of BMSCs into OS and AD were still unknown [17-19]. Thus, temporal expression profiles in the early stage of the BMSCs to differentiate into $\mathrm{OS}$ and $\mathrm{AD}$ may better understand the mechanisms of their lineage commitment determinants.

The rapid development of high-throughput sequencing provides a more systematic and comprehensive approach to studying physiological or pathological mechanisms [20]. Characterizing the features of transcriptional regulation during the different stages of stem cell development would be contributed to clarifying the mechanism of lineage commitment differentiation. Zhang et al. had firstly reported the stage-specific interaction patterns between oocyte and granulosa cells during the development of folliculogenesis with the mRNAsequencing (mRNA-Seq) technology [21]. Moreover, novel regulators of early cardiomyocyte development from human embryonic stem cells were identified with the temporal transcriptome and methylome [22]. Therefore, bioinformatics analysis is a powerful tool to discover the molecular-level biomarkers and related pathways in the osteogenic and adipogenic differentiation from BMSCs.

In the present study, we utilized the temporal gene expression profiles of an early stage in the in vitro induced (0-4 days) OS and AD from human BMSCs (hBMSCs), combined with bioinformatics and experiments, to explore the potential lineage commitment mechanisms of hBMSCs to differentiate into OS or AD. By comparing the multiple profiles, the hub-genes and key signaling pathways that critical for osteogenic and adipogenic differentiation were identified. Our work might provide the key clues for the mechanism of stepwise osteogenesis and adipogenesis, which would provide a solid reference for the prevention and therapy of osteoporosis.

\section{Materials and methods}

\section{Affymetrix microarray data source}

GSE80614, a gene expression profile of hMSCs differentiated into OS or AD based on GPL6947 platform (Illumina HumanHT-12 V3.0 expression bead chip) were downloaded from the Gene Expression Omnibus (GEO) database. It contains 66 samples including 11 groups in OS or AD differentiation and 3 samples in each group. For the differentiation of BMSCs, the first 2 days are the lineage acquisition phase and the first 2-4 days are the lineage stable phase. So samples in day 0 (0d), day 2 (2d), day 3 (3d), and day 4 (4d) groups of OS or $A D$ were selected to explore the crucial regulators involved in the initial stage of differentiation [23]. More information about the profile could be obtained from the online description (https://www.ncbi.nlm.nih.gov/geo/query/ acc.cgi?acc=GSE80614). The study protocols were reviewed and approved by the Ethics Committee of Guangxi Medical University (Nanning, China).

\section{Screening of differential expression genes (DEGs)}

All the data were normalized and analyzed with R-based web application in GEO dataset (GEO2R) [24]. The data was preprocessed with excluding the probes with no gene annotation and selecting the probes with maximum expression value if a gene is corresponding to multiple probes. DEGs were identified by the inclusion criteria 
with $P$ value $<0.05$ and fold change $(\mathrm{FC}) \geq 2$ [25]. The visualization of DEGs was drawn via volcano plot and Venn diagram by using the ggplot2 and VennDiagram packages in $\mathrm{R}$ language (version 3.5.1) (http://www.rproject.org/) [26].

\section{Function enrichment analysis of DEGs}

Gene Ontology (GO; http://www.geneontology.org/) analysis, a common and useful method for the computational analysis of large-scale molecular biology and transcriptome data in biomedical research, can classify the genes into three functional categories, including the biological process (BP), cellular component (CC), and molecular function (MF) [27, 28]. Kyoto Encyclopedia of Genes and Genomes (KEGG, http://www.genome.jp/ $\mathrm{kegg} /$ pathway.html) is a well-knowledge database for understanding the molecular interaction and relation networks [29]. To expound the distinctive biological function, the identified DEGs were utilized to perform the GO term and KEGG pathway enrichment analysis with the database for annotation, visualization, and integrated discovery (DAVID v6.8, https://david.ncifcrf.gov/ tools.jsp) online tool [30]. The cutoff value of the result was set at $P$ value $<0.05$. Scatter plots were used to depict the top 10 and command KEGG pathways in the Image GP (http://www.ehbio.com/ImageGP/), and GO term enrichment analyses were visualized via the bar plot and cord plot with GOplot package in R [31].

\section{Construction of protein-protein interaction (PPI) networks} Search Tool for the Retrieval of Interacting Genes (STRI NG, v.10.5; http://string-db.org/cgi/input.pl), an online biological database and web resource, provides integrations and assessments of the PPI which could assess the relationships between DEGs [32]. Score (median confidence) > 0.4 was set as the cutoff criterion of the selected PPIs, followed by PPI network construction via Cytoscape software (version 3.4.0; http://www.cytoscape. org/). The PPI networks, in which each node represents a protein and the lines denote direct interactions between proteins, were then used Between and Stress algorithm of cytoHubba in the Cytoscape to screen the top 20 hub genes in the OS and AD DEGs [33].

\section{Cell isolation and culture}

The hMSCs were isolated from the marrows' fluid extracted from the patients with traumatic joint replacement based on informed consent. The cells were cultured in Dulbecco's modified Eagle's medium (DMEM) containing 10\% FBS, penicillin $(100 \mathrm{U} / \mathrm{ml})$, and streptomycin $(100 \mathrm{mg} / \mathrm{ml})$ underling $37^{\circ} \mathrm{C}$ and $5 \% \mathrm{CO}_{2}$. The medium was replaced every 2 days, and the cells at passage 2 were used for further studies.

\section{Induction of hBMSCs into $O S$ and $A D$}

Prior to induction, hMSCs with a density of $1.5 \times 10^{4}$ / $\mathrm{cm}^{2}$ were seeded in growth medium until reach to 90 $100 \%$ confluence. Then, the medium was changed with the OS or AD induction medium for 4 days. The OS induction medium was prepared with $\alpha$-MEM that supplemented with $10 \mathrm{mmol} / \mathrm{L}$ of glycerol 2-phosphate, 100 $\mathrm{nmol} / \mathrm{L}$ of dexamethasone, and $50 \mu \mathrm{mol} / \mathrm{L}$ of ascorbic acid. For AD induction medium, $5 \mu \mathrm{g} / \mathrm{ml}$ insulin, $1 \mu \mathrm{M}$ dexamethasone, $500 \mu \mathrm{M}$ 3-isobutyl-1-methylxantine and $50 \mu \mathrm{M}$ indomethacin were added into DMEM [34].

RNA extraction and quantitative real-time PCR (qRT-PCR) Intracellular total RNA of the induced samples was purified via a Hipure Total RNA Mini kit (Magen, China) after $0,2,3$, and 4 days of induction. Next, $1000 \mathrm{ng}$ extracted RNA was reverse transcribed to cDNA using a cDNA synthesis kit (Takara, China). A LightCycler ${ }^{\circledR} 480$ Sequence Detention System (Roche, Germany) with PCR Green Master Mix (Roche, Germany) was used to conduct the qRT-PCR for the detection of gene expression. Forkhead box O3 (FoxO3), phosphoinositide-3-kinase regulatory subunit 1(PIK3R1), catalase (CAT), interleukin 6 (IL6), alkaline phosphatase (ALP, an early maker of OS), vascular endothelial growth factor $\mathrm{A}$ (VEGFA), fibroblast growth factor 2 (FGF2), and lipoprotein lipase (LPL, an early marker of $\mathrm{AD}$ ) were normalized by glyceraldehyde-3phosphate dehydrogenase (GAPDH) as a control. Table 1 lists the primer sequences of the above genes.

Table 1 Primer sequences used in qRT-PCR experiments

\begin{tabular}{ll}
\hline Primer & Sequence(5' to $^{\prime}$ ) \\
\hline ALPP-F & CCAGATGACTACAGCCAAGG \\
ALPP-R & GAGTCTCGGTGGATCTCGTAT \\
PIK3R1-F & TAGTGGTGGACGGCGAAGTA \\
PIK3R1-R & TGGAGGAGTCGTTGTGCTG \\
FoxO3-F & AGTCGGACCCCTTGATGTCT \\
FoxO3-R & GGTGGTGGAGCAAGTTCTGAT \\
IL6-F & GTAGTGAGGAACAAGCCAGAGC \\
IL6-R & GTTGGTCAGGGGTGGTAT \\
CAT-F & AGATAGCCTTCGACCCAAGC \\
CAT-R & AGCACGGTAGGGACAGTTCA \\
LPL-F & GGCTGGACGGTAACAGGAAT \\
LPL-R & CAGCCAGTCCACCACAATGA \\
VEGFA-F & GAAGGAGGAGGGCAGAATCA \\
VEGFA-R & GGTCTCGATTGGATGGCAGT \\
FGF2-F & AGAGCGACCCTCACATCAAG \\
FGF2-R & AGCCAGGTAACGGTTAGCAC \\
\hline
\end{tabular}




\section{Immunofluorescence staining}

The expression levels of proteins in the key signaling pathway were detected by immunofluorescence. The cells were removed from the well plate and cleared three times with PBS. After fixed with $4 \%$ (w/v) paraformaldehyde for $20 \mathrm{~min}$, the cells were transparented in the Triton $\mathrm{X} 100$ for $15 \mathrm{~min}$. Subsequently, endogenous peroxidase activity was blocked by the $\mathrm{H}_{2} \mathrm{O}_{2}$ for 15 min at $25{ }^{\circ} \mathrm{C}$. Then, the cells were incubated with primary antibodies containing PIK3R1 (1:500), FoxO3 (1:100), CAT (1:100), IL6 (1:100), VEGFA (1:200), and FGF2 (1: 100) at $4{ }^{\circ} \mathrm{C}$ for $12 \mathrm{~h}$ according to reagent instructions. After added with secondary antibodies for $1 \mathrm{~h}$ at $37^{\circ} \mathrm{C}$, the cells were observed and photographed by using an inverted phase-contrast microscope (OLYMPUS, Japan).

\section{Statistical analysis}

Statistical analysis of the data was performed by one-way ANOVA and the quantitative data were expressed as means \pm standard deviation of the experiment. $P<0.5$ was set as the cutoff of significant differences.

\section{Results}

\section{DEGs and KEGG pathway of OS induction}

To systematically explore gene expression changes during the early stage of hMSCs differentiated into OS, we identified the DEGs by comparing the OS_2d, OS_3d,
OS_4d groups with the group OS_0d. There were 452 DEGs in the OS_2d group, including 262 upregulations and 190 downregulations while 656 DEGs in the OS_3d group, of which 350 were upregulated and 306 were downregulated (Fig. 1a, b). In addition, a total of 687 genes with 378 upregulations and 309 downregulations were detected to be differentially expressed in the OS $4 \mathrm{~d}$ group (Fig. 1). Moreover, the common upregulations were nearly twice compared to downregulations in these three time points, which might mean that the OS was the result of the upregulation of pro-differentiation genes and activation of related pathways (Figure S1A and $\mathrm{B})$. To explore the potential mechanisms in the OS initiative differentiation, KEGG pathway enrichment analysis was performed with DAVID database. The top 10 pathways sorted by the number of DEGs enriched in every term were displayed via scatter plot (Fig. 1d-f). Obviously, PI3K-Akt signaling pathway was the most significant pathway in each time point during the OS differentiation.

\section{DEGs and KEGG pathway of AD induction}

There were a total of 729 DEGs in AD_2d group, 870 DEGs in AD_3d group, and 905 DEGs in AD_4d group, including 364, 441, and 461 upregulations while 365, 429 , and 444 downregulations in the AD_2d, AD_3d, and $A D \_4 d$ group, respectively (Fig. $2 \mathrm{a}-\mathrm{c}$ ). The count of

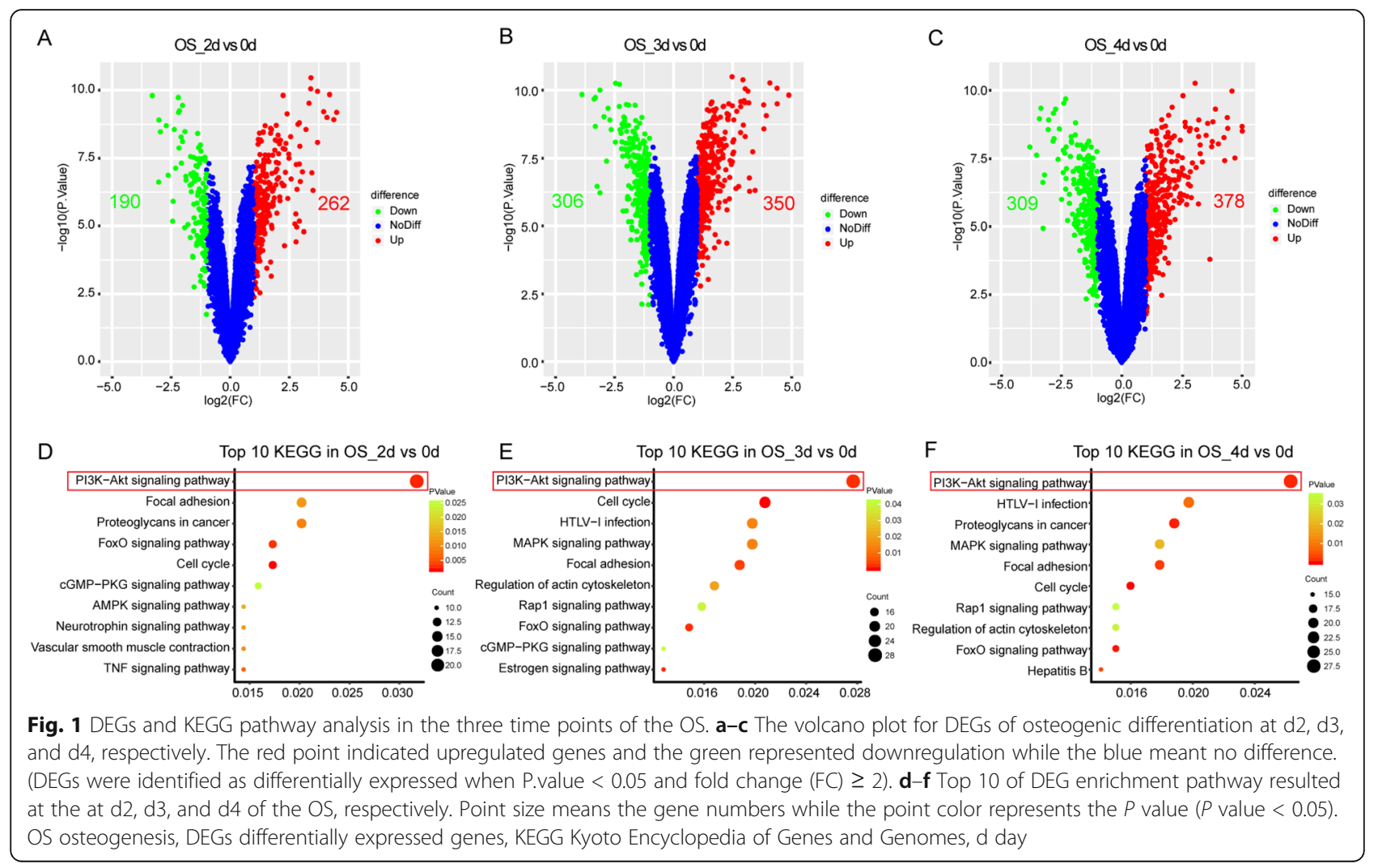




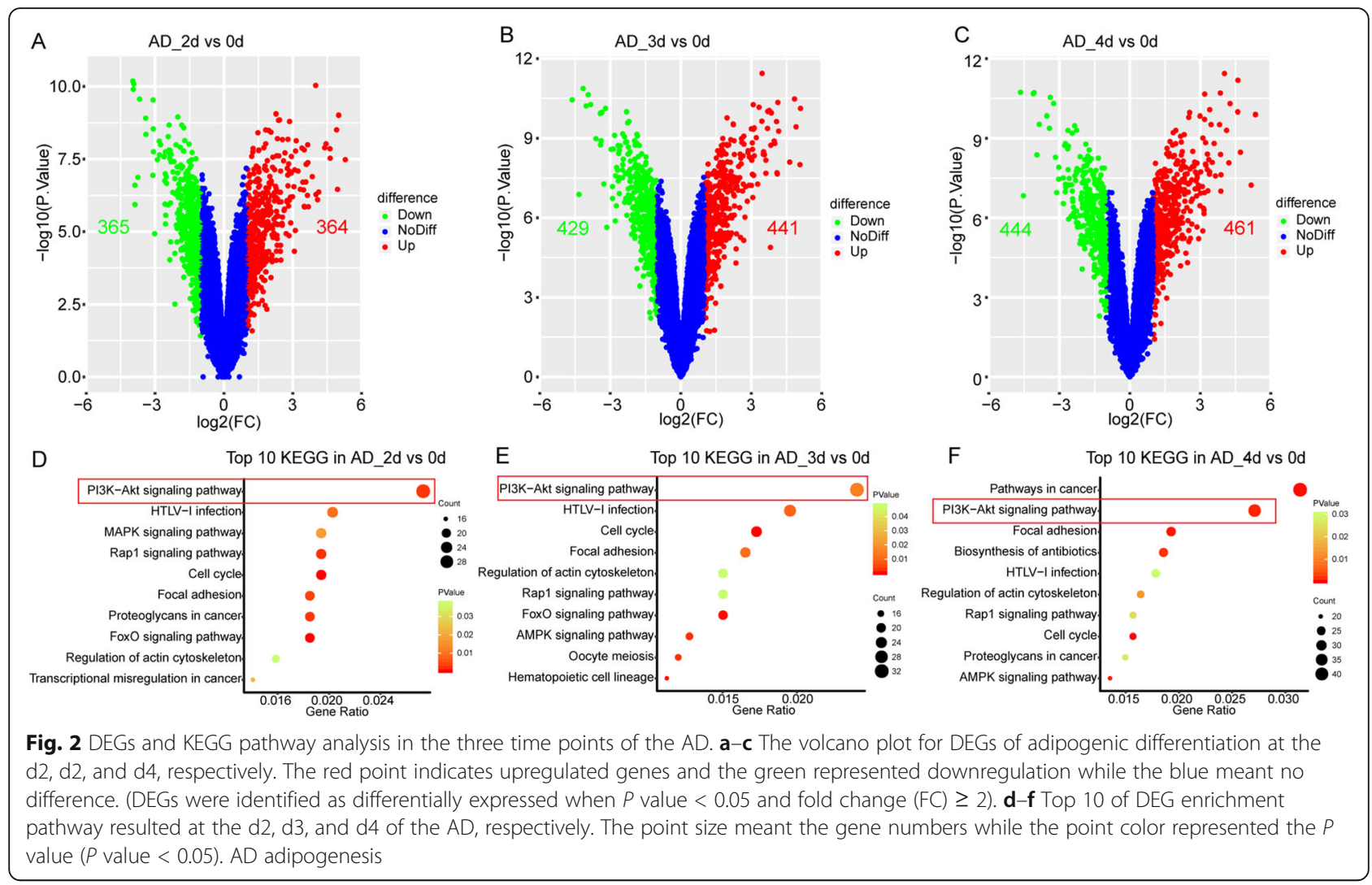

DEGs in AD induction showed little change in each time point as well, but increased significantly compared to DEGs OS, which might be due to hMSCs favor differentiate into $\mathrm{AD}$ rather than $\mathrm{OS}[35,36]$. As shown in the Venn diagrams, the number of the downregulations was similar to the upregulations in the $\mathrm{AD}$, but was more than twice that in the OS, meaning the downregulation play more significance in the AD (Figure S1A and B). As the same as OS induction, the top 10 KEGG pathway enrichment at each time point was visualized in the scatter plot (Fig. 2d-f). According to numbers of enriched DEGs, the PI3K-Akt signaling pathway was also ranked at first, ignoring the pathways in cancer usually enriched most genes in the DAVID. Therefore, we surmised that the PI3K-Akt signaling pathway also exerted a crucial effect on AD from BMSCs.

\section{Key KEGG pathway enrichment}

We next investigated the key signaling pathway in these two lineages of differentiation. The PI3K-Akt signaling pathway, FoxO signaling pathway, focal adhesion, and cell cycle were the common signaling pathways in OS differentiation (Fig. 3a). In AD induction, PI3K-Akt signaling pathway, Rap1 signaling pathway, focal adhesion, regulation of actin cytoskeleton, cell cycle, and HTLV-I infection were all enriched in the three groups (Fig. 3c). Three common pathways including PI3K-Akt signaling pathway, focal adhesion, and cell cycle were all existed in the OS and AD induction. Therefore, we hypothesized that the PI3K-AKT signaling pathway, as well as focal adhesion, and cell cycle were the crucial mechanism for initiating the differentiation of hMSCs. For the differences between the two lineage differentiations, the FoxO signaling pathway was specific for OS induction and the Rap1 signaling pathway belonged only to AD induction, indicating that FoxO signaling pathway and Rap1 signaling pathway were the specific signaling pathway for the $\mathrm{OS}$ and $\mathrm{AD}$ induction, respectively. And the expression level of DEGs enriched in the FoxO signaling pathway and Rap1 signaling pathway were showed at heatmap (Fig. 3b, d).

\section{Key genes in the OS induction}

Interactions between multiple genes could be well understood by PPI analysis. The DEG OS was used to perform the PPI analysis with Between and Stress algorithm of cytoHubba, from which we choose the top 20 hub genes to construct the sub-network (Fig. $4 \mathrm{a}-\mathrm{c}$ and Figure S2). As shown in these six PPI networks, the common genes with high degree and clustering coefficients were FoxO3, IL-6, JUN, and CAT, which might play important roles in the OS induction. At the same time, candidate genes enriched in the FoxO signaling pathway in each group were also used to perform the 


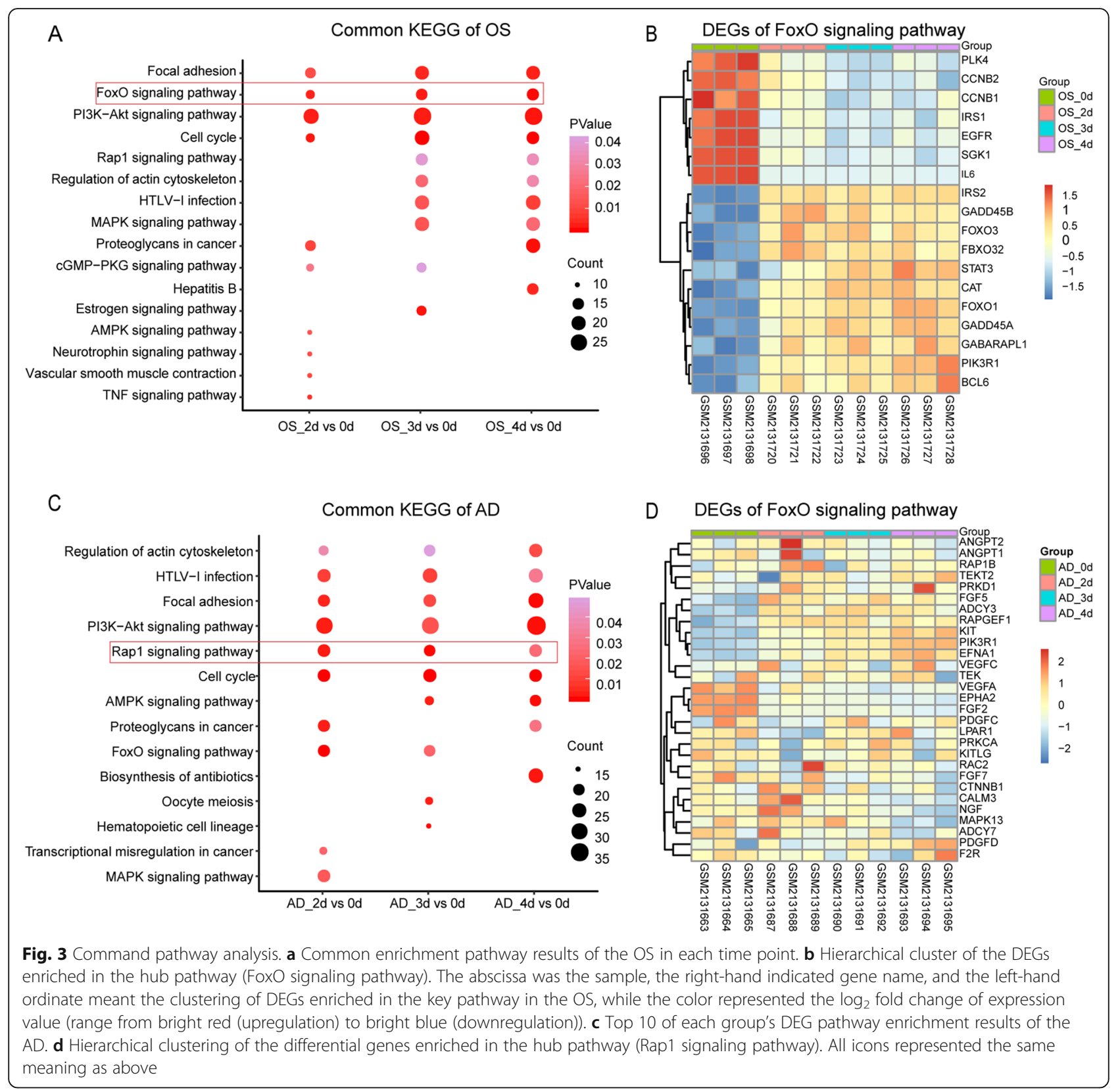

PPI network (Fig. 4d-f). Similarly, FoxO1, IL-6, and CAT were localized at the center of the network. These results indicated that FoxO3, IL-6, JUN, and CAT may be the key genes for early OS differentiation. Since FoxO1, IL-6, and CAT were all enriched in the FoxO signaling pathway, it is further indicating that FoxO signaling pathway may be the specific signaling pathway for early OS differentiation.

\section{Key genes in the AD induction}

DEGs AD was used to perform the PPI networks, and sub-networks were formed by the top 20 hub genes. VEGFA, FGF2, MYC, and PTEN had the highest degree and clustering coefficients in each group computed by Between algorithm (Fig. 5a-c), while VEGFA, FGF2, MYC, CCND1, and PTEN were the core computed by Stress algorithm (Figure S3), which meant that these genes played core roles in the AD induction. Similarly, the DEGs related to Rap1 signaling pathway were selected to construct PPI network. VEGFA, FGF2, and PIK3R1 were identified as the core genes in the network (Fig. 5d-f). These interactions relied strongly on VEGFA and FGF2, which may determine the tendency of BMSCs to differentiate into AD. Therefore, hub genes VEGFA, FGF2, and Rap1 signaling pathway might be the crucial mechanisms for early AD differentiation. 


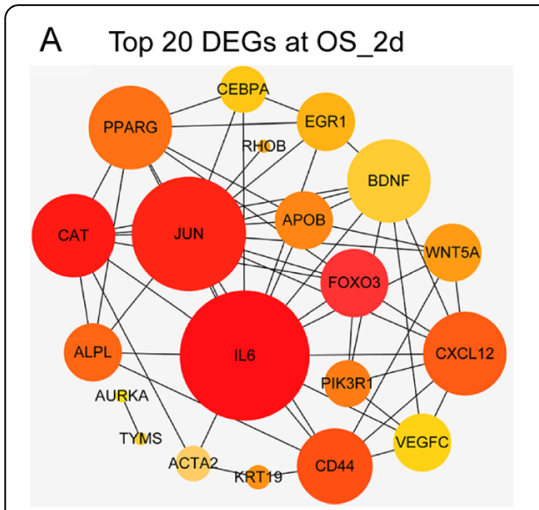

D DEGs enriched in FoxO at OS_2d

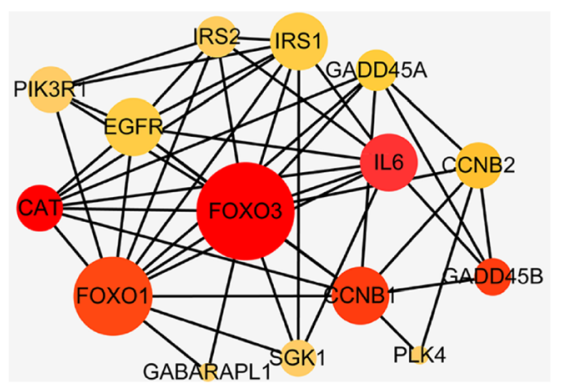

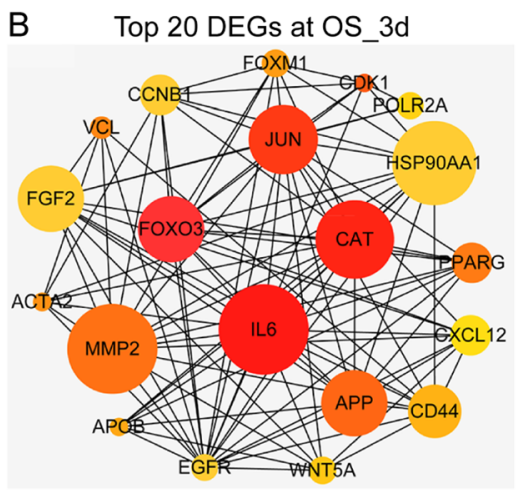

E DEGs enriched in FoxO at OS_3d

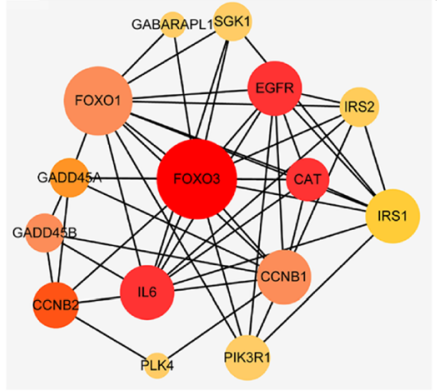

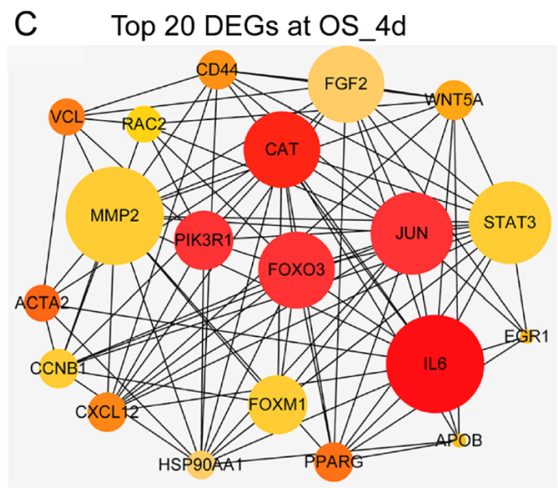

F DEGs enriched in FoxO at OS_4d

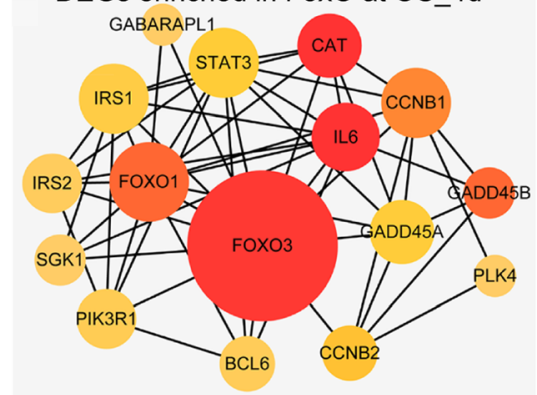

Fig. 4 Key gene analysis in the OS. a-c Network representation of the protein-protein interactions (PPI) between the top 20 genes of the DEGs in different time points in the OS by the Between mode using cytoHubba app in the Cytoscape. The node's colors donated the clustering coefficient, and the brighter the color, the higher the clustering, which meant stronger interaction (range from red (high) to yellow (low)), while the size of each node represented node degree distribution. $\mathbf{d}$-f PPI visualization of networks with the DEGs enriched in the hub pathway (FoxO signaling pathway) of the OS, the color, and size of each point meant as the same meaning as above. $\mathbf{a}, \mathbf{d} 2 \mathrm{~d}$ vs $0 \mathrm{~d}$; $\mathbf{b}$, e $3 \mathrm{~d}$ vs $0 \mathrm{~d}$; c, f $4 \mathrm{~d}$ vs $0 \mathrm{~d}$

\section{GO analysis of OS induction}

Gene ontology enrichment analyses were performed by the DEGs from each group, and the significant top 15 of the GO terms including BP, CC, and MF were displayed as the bar diagrams (Fig. 6a-c). In the MF category, receptor binding, protein binding, and growth factor activity were the common GO terms in the groups of OS_2d, OS_3d, and OS_4d, while cytoplasm, cytosol, and extracellular exosome were the common GO terms in the CC category. In the BP category, signal transduction, positive regulation of transcription, and positive regulation of cell proliferation were all enriched in the three time points of OS induction. To further investigate the relationship of the key genes and GO functional categories, the key genes from the FoxO signaling pathway and PI3K-Akt signaling pathway were put to perform the chord plots (Fig. 6d-f). Key genes including FoxO3, IL6, CAT, and PIK3R1 mainly clustered into protein binding, membrane, cytosol, nucleus, extracellular space, plasma membrane, cytoplasm, negative, and positive regulation of the apoptotic process. Suggesting that GO terms in OS induction were a series of biological responses that initiated by ligand-receptor binding and transcriptional information into the nucleus.

\section{$G O$ analysis of $A D$ induction}

The top 15 of the GO terms performed by the DEGs from each group of $\mathrm{AD}$ were shown in the bar diagrams (Fig. 7ac). The results of the $\mathrm{AD}$ differentiation were similar to that in the OS, implied that there were similar biological effects on the two lineage differentiations from hMSCs. Chord plots of $\mathrm{AD}$ induction (Fig. $7 \mathrm{~d}-\mathrm{f}$ ) performed by the DEGs of Rap1 signaling pathway and top 15 of GO terms in each group, showed that FGF2 was mainly enriched in the protein binding, growth factor activity, cytoplasm, nucleus, extracellular space, extracellular region, signal transduction, positive regulation of cell proliferation, and positive regulation of cell proliferation. Moreover, another target gene VEGFA was mainly related to protein binding, growth factor activity, membrane, extracellular space, extracellular region, cytoplasm, and positive regulation of cell proliferation. This was consistent with previously reported that VEGFA was an extracellular signal molecule, which regulated Rap1 signaling pathway by combining some intracellular signal factors [37, 38].

\section{Osteo-adipogenesis relative gene expression}

The expression level of OS (ALP) and AD (LPL) specific genes at days 2-4 was increased compared to day 0 , suggesting that hBMSCs were successfully induced into OS 


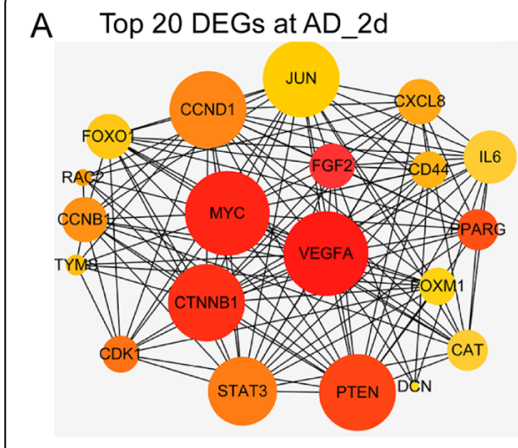

D DEGs enriched in Rap1 at $A D \_2 d$

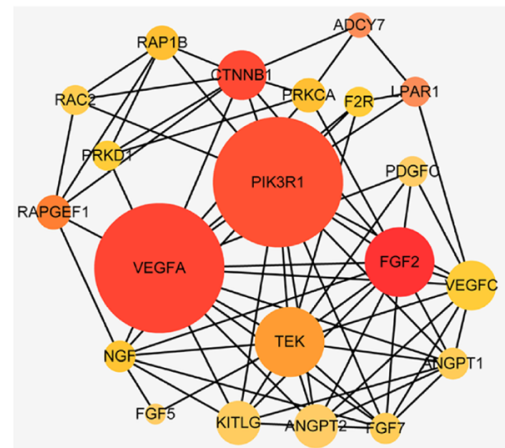

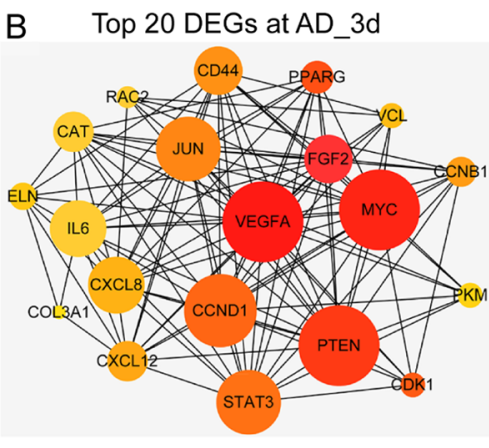

E DEGs enriched in Rap1 at AD_3d

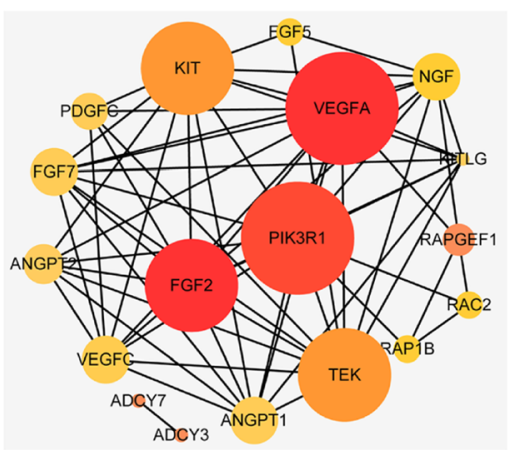

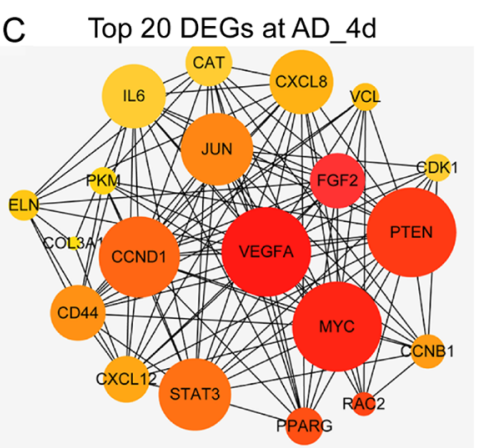

F DEGs enriched in Rap1 at $A D \_4 d$

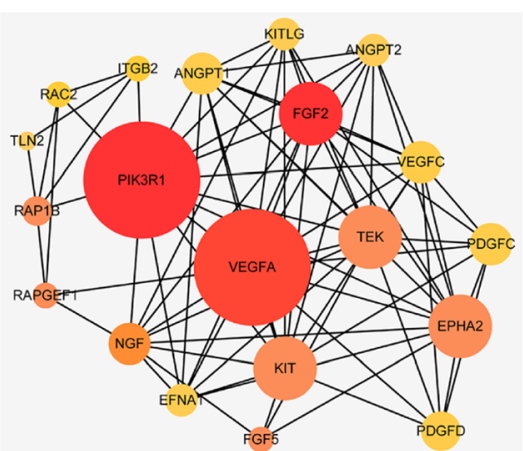

Fig. 5 Key genes analysis in the AD. a-c Network representation of the protein-protein interactions (PPI) between the top 20 genes of the DEGs in different time points in the AD by the Between mode using cytoHubba app in the Cytoscape. The node's colors donated the clustering coefficient, and the brighter the color, the higher the clustering, which meant stronger interaction (range from red (high) to yellow (low)), while the size of each node represented node degree distribution. $\mathbf{d}-\mathbf{f}$ PPI visualization of networks with the DEGs enriched in the hub pathway (Rap1 signaling pathway) of the AD, the color, and size of each point meant as the same meaning as above. a, $\mathbf{d} 2 \mathrm{~d}$ vs $0 \mathrm{~d}$; $\mathbf{b}$, e $3 \mathrm{~d}$ vs $0 \mathrm{~d}$; c, $\mathrm{f} 4 \mathrm{~d}$ vs $0 \mathrm{~d}$

and $\mathrm{AD}$ (Fig. 8a, c). The expression levels of the key genes for OS (IL6 and CAT) or AD (PIK3R1, FGF2, and VEGFA) induction were changed dramatically at the first 2 days, but no obvious change was founded after that, indicating that the first 2 days may be the critical period for the lineage commitment determinant. However, FoxO3 at days $2-4$ was significantly increased in the OS induction, revealing that it may be key genes for osteogenic differentiation at a steady period of lineagecontinuation (Fig. 8a). In addition, the expression of PIK3R1 was higher in both OS and AD, suggesting PIKAKT signaling pathway is a promoter in the differentiation of BMSCs.

\section{Signaling pathway of $O S$ and $A D$ induction}

The expression of the key proteins in the signaling pathway relative to the $\mathrm{OS}$ and $\mathrm{AD}$ induction were detected using inflorescence staining (Fig. 8b, d). In consistent with the results of PCR, the expression of FoxO3 and CAT in OS induction at days 2-4 was higher compared to day 0 , while IL6 was conversely lower, suggesting that FoxO3 signaling pathway was involved in the osteogenic differentiation (Fig. 8b). Additionally, the downregulation of VEGFA and FGF2 in AD induction indicated that
Rap1 signaling pathway may be crucial for AD differentiation. Meanwhile, the expression of PIK3R1 protein was also increased both in OS and AD.

\section{Discussion}

Loss of bone mass and aggravation of micro-fractures increase the bone fragility and susceptibility to fractures in patients of osteoporosis [39]. Currently, most strategies involving drugs based on inhibiting osteoclast activity and then blocking bone resorption were reported to cause serious adverse reactions. For example, denosumab, a new monoclonal antibody targeting RANKL, is an effective treatment of osteoporosis, but its serious side-effects including increased risk of spinal fracture and osteonecrosis, and the rebound effect after stopping denosumab exposure limit its clinical application [4043]. Thus, there is currently a lack of enough safe and effective treatment strategy for dealing with this gap [44, 45]. Many studies have confirmed that the pathogenesis of osteoporosis is highly correlated with the tendency of increased adipocytes and decreased osteoblasts [46-48], and our analysis found the number of the DEGs in the $\mathrm{AD}$ was more than that of OS corresponding to previous studies (Figs. 1 and 2). Therefore, clarifying the detailed 


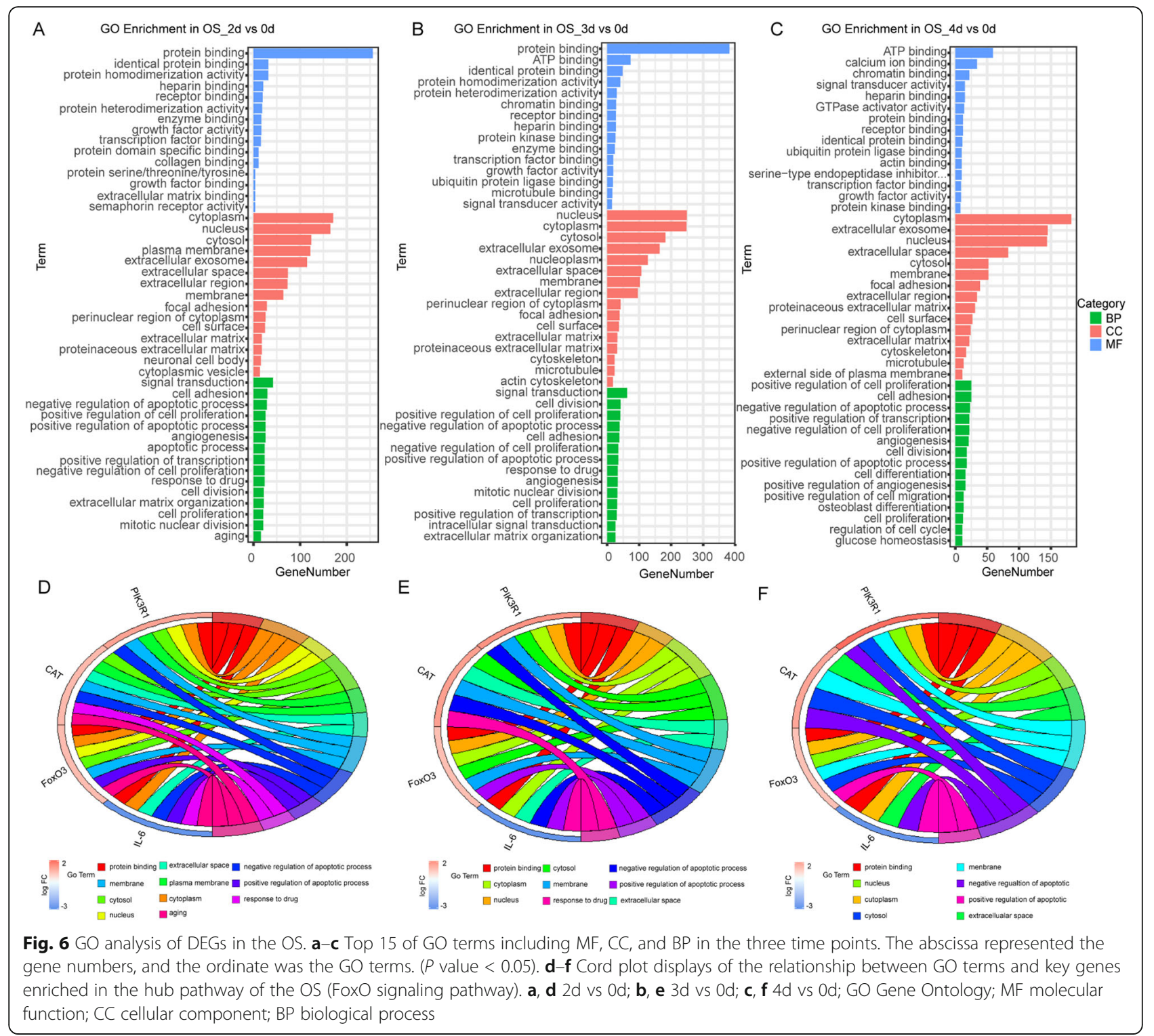

mechanisms about the differentiation of MSCs into OS and $\mathrm{AD}$ would be contributed to develop a new strategy and treatment for osteoporosis. As the differentiation of MSCs into a specific lineage was determined at the early stage of differentiation $[49,50]$, we selected early OS and $\mathrm{AD}$ profiles to explore the early differentiation mechanisms that determined lineage fates of BMSCs.

After a series of integration analyses, we identified FoxO3, IL-6, and CAT as key genes for osteogenic differentiation, and these genes were all enriched in the FoxO signaling pathway (Fig. 4 and S2). FoxO, an intracellular signaling factor belongs to transcriptional regulators family of forkhead box O, consisting of FoxO1, FoxO3, FoxO4, and FoxO6, plays a critical regulatory role in the multiple biological processes, including cell cycle, anti-apoptosis, and anti-oxidation [51, 52].
Previous studies had discovered that FoxO3 could reduce ROS and promote OS differentiation of MSCs by activating autophagy [53-55], which demonstrated that the upregulation of FoxO had an essential role on the activity of the OS in MSCs. Wen Sun had verified that Sirt1 overexpression promoted FoxO3a deacetylation and inhibited oxidative stress and resisted the apoptosis to increase the osteogenesis and partially restoring the defects of the skeletal system in osteoporosis [56]. However, lots of evidences also indicated that FoxO3 has a contradictory function in different tissues or expression levels. Anthony et al. had reported that the overexpression of FoxO3 could result in muscle atrophy while the ablation of FoxO3 may lead to defects in the regeneration of the muscle by downregulating MyoD (a key myogenic regulator) [57-59]. Meanwhile, Lorenowicz 


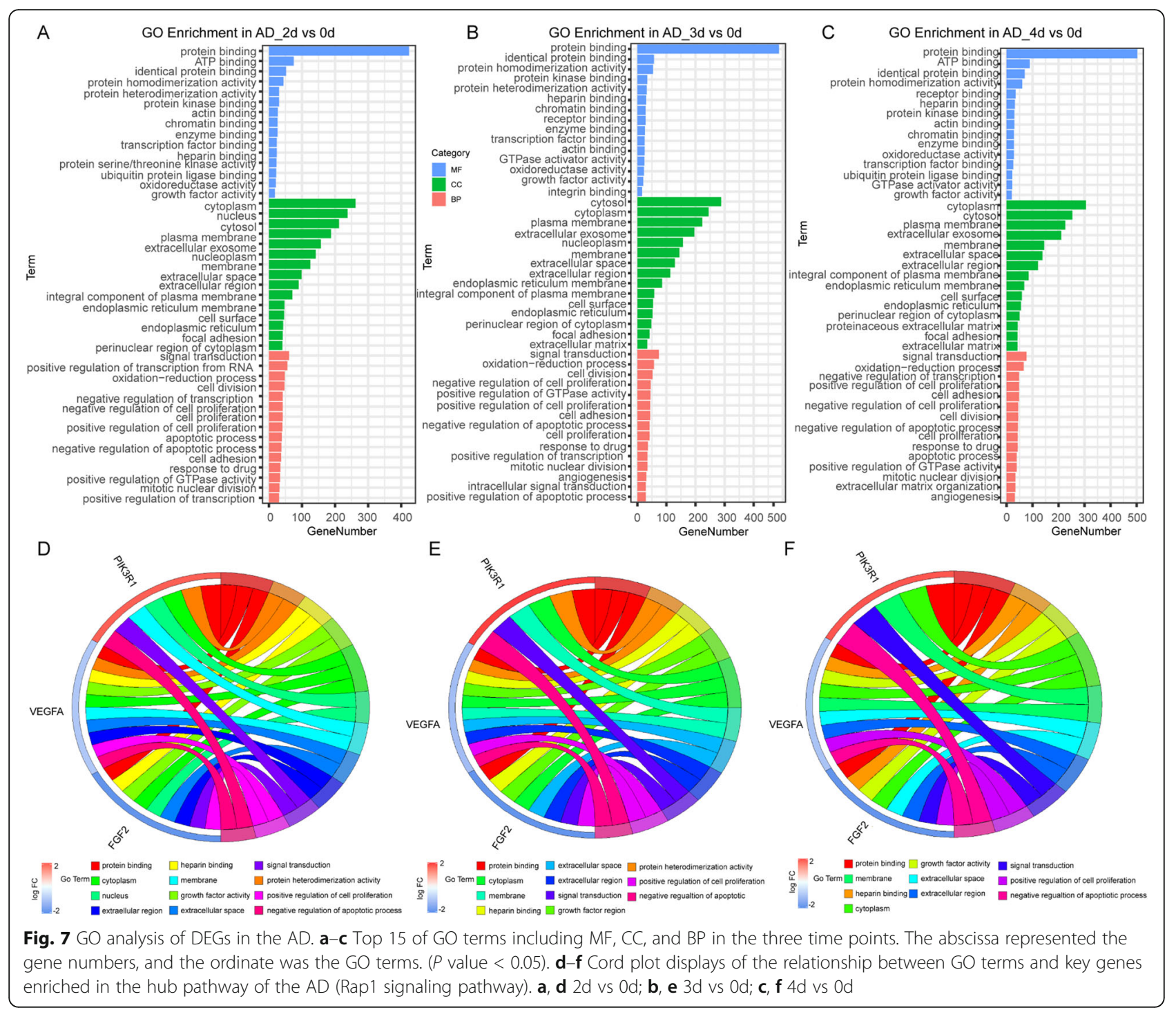

et al. have confirmed that activation of autophagy accelerated the osteogenic differentiation via the upregulation of FoxO3, consistent with our findings. On the contrary, stem cell lost a capacity to avoid rising ROS and osteogenic differentiation was impaired on the FoxO3 downregulation [60]. Therefore, we speculate that the function of FoxO may be dependent on the activation level and tissue location, of course, which requires further basic and animal experiments, and even future clinical experiments to discover the mechanism of the contradictory functions, revealing whether the activation of FoxO3 would bring other side effects when promoting OS in BMSCs, such as skeletal muscle atrophy. IL-6, a pleiotropic cytokine with multiple physiological functions including immune regulation, hematopoiesis, and tissue regeneration, plays a paramount role in the tissue regeneration engineer, especially in the bone metabolism $[61,62]$. It could stimulate the hepatocytes to return to the cell cycle progression through triggering the initiative signal of liver regeneration [63]. Research showed that HIF1A-AS2 could erase the antagonistic effect of IL- 6 exerted by miR-665 and then promote osteogenic differentiation of the BMSCs [64]. Zhongyu Xie also confirmed that IL-6 had an important role in the osteogenic differentiation of BMSCs [65]. As for CAT, Mao Li had illuminated that it could promote osteogenic differentiation through enhancing resistance to oxidative stress [66]. Next, we extracted the expression value of FOXO3, IL-6, and CAT (Fig. 8a), suggesting FOXO3 and CAT were positively correlated with osteogenic differentiation, while IL- 6 was negative. Based on the previous studies, we surmised that FOXO3, IL-6, and CAT were key genes in the OS and FoxO was a core signaling pathway, especially in the early stage of OS.

In the $\mathrm{AD}$, we identified hub-genes of VEGFA and FGF2 in the Rap1 signaling pathway was the essential 


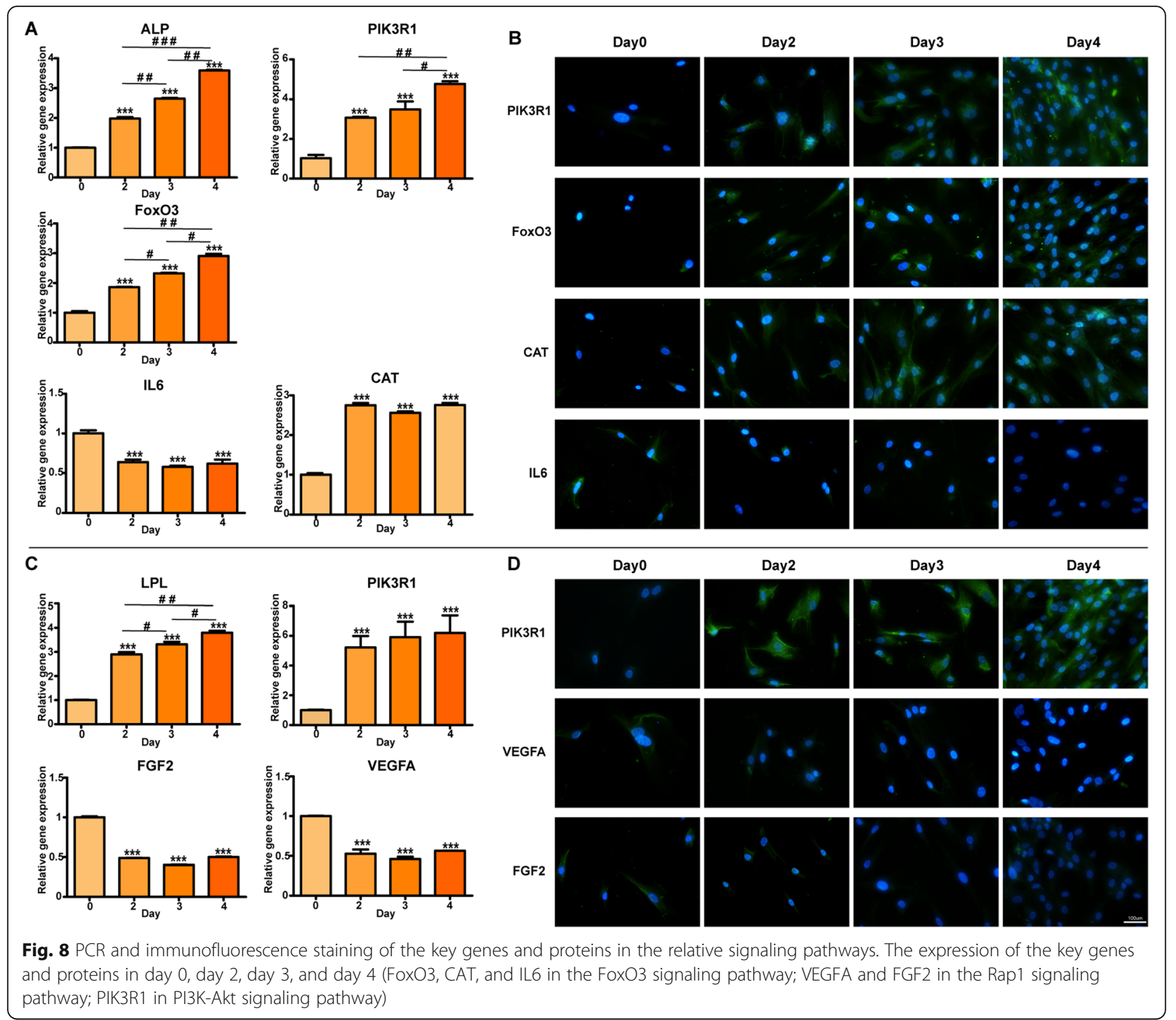

mechanisms involved in the early stage of adipogenic differentiation (Fig. 5 and S3). Frank Yeung had indicated that Rap1, a mammalian telomere-binding protein, played a key role in the AD through its additional nontelomeric functions, which was known as co-factor of transcriptional cascade and regulator of NF-kB pathwa $y[67]$. VEGFA, a key angiogenic factor, was initially considered as an important molecular in the angiogenesis, and latterly was identified that it had multiple biological functions including bone cellular survival [68]. Wen Zhang pointed out that miR-128 promoted the adipogenic differentiation of hMSCs by the suppression of the VEGF pathway [69]. As a heparin-binding growth factor stored in the extracellular matrix, FGF2 had been identified to be an important modulator in the early differentiation and development of cells, owning to its multiple biological functions including chemotaxis, angiogenesis, and mitotic activity [70]. Kim et al. [71] had found that BMSCs with a deficiency of FGF2 showed a strong capacity of adipogenic differentiation, which indicated that downregulated FGF2 played an important role in the AD. Thus, VEGFA and FGF2 were both negative factors during adipogenic differentiation, which was consistent with our analysis (Fig. 8b). In the AD, FGF2 might increase the conduction of extracellular signals through molecular adhesion and then regulate the Rap1 signaling pathway to promote the differentiation of BMSCs.

In the trigger phase of BMSC differentiation, we speculated PI3K-Akt signaling pathway played a key role in triggering the differentiation of stem cells into various progenitor lines, at least in the OS and $\mathrm{AD}$, based on the previous studies and our surveys (Fig. 1 and $2 \mathrm{~d}-\mathrm{f}$ and Fig. 3a-c). As shown in our analysis, the PI3K-Akt signaling pathway enriched the most DEGs during the 
whole process of the early stage in OS and AD (Figs. $1 d-f$ and $2 d-f)$, which meant a paramount role in the differentiation of BMSCs. Activation of PI3K-Akt signaling pathway had been demonstrated not only to promote the $\mathrm{OS}$ and $\mathrm{AD}$, but also to stimulate the chondrogenic differentiation of BMSCs, while its antagonist could lead to inhibition of BMSC differentiation [72-74]. Through the PI3K-Akt signaling pathway, BMSCs can also differentiate into other progenitor cells, such as endothelial cells (ECs) and vascular smooth muscle cells $[75,76]$. Researches had confirmed that stimulation of PI3K-Akt signaling pathway had the ability to activate its downstream target of rapamycin-p70S6 kinase and promote BMSCs to differentiate into coulvascular smooth muscle. Proangiogenic bioscaffold composited of porous $\beta-\mathrm{CaSiO}(3) / \mathrm{PDLGA}$ advanced the EC differentiation of BMSCs via activating PI3K-Akt signaling pathway, which in turn promoted phosphorylation of endothelial nitric oxide synthase (eNOS), production of nitric oxide (NO), and increased secretion of vascular endothelial growth factor (VEGF). Therefore, we surmised activation of PI3K-Akt signaling pathway in the trigger phase of BMSC differentiation might subsequently activate the FoxO and Rap1 signaling pathways, respectively, thereby promoting $\mathrm{OS}$ and $\mathrm{AD}$. And the expression level of the key gene PIK3R1 from PI3K-Akt signaling pathway was upregulated both in the OS and $\mathrm{AD}$, which are in line with our speculation (Fig. 8a, b).

Based on the GO enrichment analysis of OS, protein binding, transcription factor binding, nucleus, membrane, cytosol, and cytoplasm were clustered in the FoxO3 and IL-6. These aggregations were similar in the adipogenesis (Figs. $6 \mathrm{~d}-\mathrm{f}$ and $7 \mathrm{~d}-\mathrm{f}$ ). From these results, it seemed that osteogenic and adipogenic differentiations were consistent in biological processes, both through protein-protein binding and transmission of signaling molecules inside and outside the membrane.

\section{Conclusions}

Taken together, this study analyzed the gene chip expression profile of hMSC differentiation to identify the potential biomarkers and key pathways between the BMSCs into OS and AD by using the bioinformatics method. Our result indicates that FoxO signaling pathway was a key pathway in the OS, and Rap1 signaling pathway is the key pathway in the $\mathrm{AD}$, while the PI3KAkt is a key signaling pathway with the key gene PIK3R1 in the initial stage of stem cell differentiation. Moreover, FOXO3, IL-6, and CAT are suggested as the potential biomarkers in the OS as well as VEGFA and FGF2 in the AD. Our research may provide a new insight into the study of BMSC differentiation, contributing to the potential therapeutic targets of osteoporosis.

\section{Supplementary information}

Supplementary information accompanies this paper at https://doi.org/10. 1186/s13018-020-01965-3.

\begin{abstract}
Additional file 1: Figur eS1. Common up-and downregulations in each time points of OS and $A D$. (A): up of OS (B): down of OS (C): up of OS (D): down of AD. Figure S2. Key genesanalysis in the OS. (A-C)Network representation of the protein-protein interactions (PPI) between the top 20 genes of the DEGs in different time points in the OS by the Stress mode using cytoHubba app in the cytoscape. The node's colors donated the clustering coefficient, and the brighter the color, the higher the clustering, which meant stronger interaction (rang from red (high) to yellow $(\mathrm{low})$ ), while the size of each node represented node degree distribution. (A) $2 d$ vs Od; (B)3d vs 0d; (C) $4 d$ vs Od. Figure S3. Key genes analysis in the $A D$. (A-C)Network representation of the protein-protein interactions (PPI) between the top 20 genes of the DEGs in different time points in the AD by the Stress mode using cytoHubba app in the cytoscape. The node's colors donated the clustering coefficient, and the brighter the color, the higher the clustering, which meant stronger interaction (rang from red (high) to yellow (low)), while the size of each node represented node degree distribution. (A)2dvs 0d; (B)3d vs 0d; (C)4d vs $0 \mathrm{~d}$.
\end{abstract}

\section{Abbreviations}

BMSCs: Bone mesenchymal stem cells; AD: Adipogenesis; OS: Osteogenesis; DEGs: Differentially expressed genes; HBMSCs: Human bone mesenchymal stem cells

\section{Authors' contributions}

$J Z, L Z$, and PC conceived and designed the study. JW performed the primary bioinformatics analysis and was a major contributor in writing the manuscript. ZZ, BZ, and XH made substantial contributions to the data analysis, including the biological significance of hub genes and figure editing. ZL and PC were involved in the edition of the manuscript. The authors read and approved the final manuscript.

\section{Funding}

This study was financially supported by the Guangxi Key Research and Development Plan (Grant No.GuikeAD19254003) and the Guangxi Science and Technology Base and Talent Special Project (Grant No. GuikeAD17129012).

\section{Availability of data and materials}

The datasets generated and/or analyzed during the current study are available from the Gene Expression Omnibus repository, https://www.ncbi. nlm.nih.gov/geo/query/acc.cgi?acc=GSE80614).

Ethics approval and consent to participate

Ethics approval was obtained from the Ethics Committee of Guangxi Medical University (Nanning, China).

\section{Consent for publication}

Not applicable.

\section{Competing interests}

All the authors in this manuscript have read and approved the final version submitted, and there are no conflicts involved in this submission.

\section{Author details}

${ }^{1}$ Guangxi Engineering Center in Biomedical Material for Tissue and Organ Regeneration, The First Affiliated Hospital of Guangxi Medical University, Nanning 530021, China. ${ }^{2}$ Guangxi Collaborative Innovation Center for Biomedicine, The First Affiliated Hospital of Guangxi Medical University, Nanning 530021, China. ${ }^{3}$ Department of Orthopaedics Trauma and Hand Surgery, The First Affiliated Hospital of Guangxi Medical University, Nanning 530021, China. ${ }^{4}$ Guangxi Key Laboratory of Regenerative Medicine, The First Affiliated Hospital of Guangxi Medical University, Nanning 530021, China. 
Received: 14 July 2020 Accepted: 14 September 2020

\section{Published online: 23 September 2020}

\section{References}

1. Compston JE, McClung MR, Leslie WD. Osteoporosis. The Lancet. 2019;393: 364-76.

2. Chang PY, Feldman D, Stefanick ML, et al. 27-Hydroxycholesterol, an endogenous SERM, and risk of fracture in postmenopausal women: a nested case-cohort study in the Women's Health Initiative. J Bone Mineral Res. 2019;34:59-66.

3. Qaseem A, Forciea MA, McLean RM, et al. Treatment of low bone density or osteoporosis to prevent fractures in men and women: a clinical practice guideline update from the American College of Physicians. Ann Internal Med. 2017;166:818-39.

4. Black DM, Rosen CJ. Clinical practice. Postmenopausal osteoporosis. New Eng J Med. 2016;374:254-62.

5. Eastell R, Rosen CJ, Black DM, et al. Pharmacological management of osteoporosis in postmenopausal women: an Endocrine Society* Clinical Practice Guideline. J Clin Endocrinol Metabol. 2019;104:1595-622.

6. Andreopoulou P, Bockman RS. Management of postmenopausal osteoporosis. Ann Rev Med. 2015;66:329-42.

7. Ensrud KE, Crandall CJ. Osteoporosis. Annals of internal medicine. 2017; 167: Itc17-itc32.

8. Miller PD. Management of severe osteoporosis. Expert Opin Pharmacother. 2016;17:473-88.

9. $\mathrm{GY} \mathrm{S}, \mathrm{HR}, \mathrm{JJ} \mathrm{H}$, et al. Plastrum testudinis extracts promote BMSC proliferation and osteogenic differentiation by regulating Let-7f-5p and the TNFR2/PI3K/AKT signaling pathway. Cell Physiol Biochem. 2018; 47:23072318 .

10. Horowitz MC, Tommasini SM. Fat and bone: PGC-1alpha regulates mesenchymal cell fate during aging and osteoporosis. Cell Stem Cell. 2018; 23:151-3.

11. BD S, CH H, CX Z, et al. Microenvironmental views on mesenchymal stem cell differentiation in aging. J Dent Res. 2016; 95:1333-1340.

12. Huang T, Liu R, Fu X, et al. Aging reduces an ERRalpha-directed mitochondrial glutaminase expression suppressing glutamine anaplerosis and osteogenic differentiation of mesenchymal stem cells. Stem cells (Dayton, Ohio). 2017;35:411-24.

13. Meng J, Ma X, Wang N, et al. Activation of GLP-1 receptor promotes bone marrow stromal cell osteogenic differentiation through $\beta$-catenin. Stem Cell Reports. 2016:6:579-91.

14. Kokabu S, Nguyen T, Ohte S, et al. TLE3, transducing-like enhancer of split 3, suppresses osteoblast differentiation of bone marrow stromal cells. Biochem Biophys Res Commun. 2013;438:205-10

15. Bian H, Lin JZ, Li C, et al. Myocardin-related transcription factor A (MRTFA) regulates the fate of bone marrow mesenchymal stem cells and its absence in mice leads to osteopenia. Mol Metab. 2016;5:970-9.

16. Wang Q, Li H, Wang D, et al. Effect of GGCX on the differentiation function of osteoporosis bone marrow mesenchymal stem cells through regulating TGFß/smad signaling pathway. Eur Rev Med Pharmacol Sci. 2019;23:722431.

17. Ng F, Boucher S, Koh S, et al. PDGF, TGF-beta, and FGF signaling is important for differentiation and growth of mesenchymal stem cells (MSCs): transcriptional profiling can identify markers and signaling pathways important in differentiation of MSCs into adipogenic, chondrogenic, and osteogenic lineages. Blood. 2008;112:295-307.

18. Hung SC, Chang CF, Ma HL, et al. Gene expression profiles of early adipogenesis in human mesenchymal stem cells. Gene. 2004;340:141-50.

19. Piek E, Sleumer LS, van Someren EP, et al. Osteo-transcriptomics of human mesenchymal stem cells: accelerated gene expression and osteoblast differentiation induced by vitamin D reveals c-MYC as an enhancer of BMP2-induced osteogenesis. Bone. 2010;46:613-27.

20. $\mathrm{XH}, \mathrm{HC}, \mathrm{D} \mathrm{H}$, et al. Mapping human pluripotent stem cell differentiation pathways using high throughput single-cell RNA-sequencing. Genome Biol. 2018; 19:47.

21. $Y Z, Z Y, Q Q$, et al. Transcriptome landscape of human folliculogenesis reveals oocyte and granulosa cell interactions. Mol Cell. 2018; 72:1021-1034. e1024.

22. Fu K, Nakano H, Morselli M, et al. A temporal transcriptome and methylome in human embryonic stem cell-derived cardiomyocytes identifies novel regulators of early cardiac development. Epigenetics. 2018;13:1013-26.
23. J vdP, T S, J T, et al. Identification of three early phases of cell-fate determination during osteogenic and adipogenic differentiation by transcription factor dynamics. Stem Cell Reports. 2017; 8:947-960.

24. J D, MA G, Bioinformatics DGJ. shinyGEO: a web-based application for analyzing gene expression omnibus datasets. Bioinformatics. 2016; 32:36793681.

25. M Z, L J, reviews ZYJSC, et al. circRNA expression profiles in human bone marrow stem cells undergoing osteoblast differentiation. Stem Cell Rev Rep. 2019; 15:126-138

26. Cai $P$, Jiang $T$, Li B , et al. Comparison of rheumatoid arthritis (RA) and osteoarthritis (OA) based on microarray profiles of human joint fibroblastlike synoviocytes. Cell Biochem Funct. 2019;37:31-41.

27. The Gene Ontology Consortium. Expansion of the Gene Ontology knowledgebase and resources. Nucleic Acids Res. 2017;45:D331-8.

28. Thomas PD, Hill DP, Mi H, et al. Gene Ontology Causal Activity Modeling (GO-CAM) moves beyond GO annotations to structured descriptions of biological functions and systems. Nat Genet. 2019;51:1429-33.

29. M K, research GSJNa. KEGG: Kyoto encyclopedia of genes and genomes. Nucleic Acids Res. 2000; 28:27-30

30. G D, BT S, DA H, et al. DAVID: Database for annotation, visualization, and integrated discovery. Genome Biol. 2003; 4:P3.

31. W W, F S-C, Bioinformatics RMJ. GOplot: an R package for visually combining expression data with functional analysis. Bioinformatics. 2015; 31: 2912-2914.

32. D S, A F, M K , et al. The STRING database in 2011: functional interaction networks of proteins, globally integrated and scored. Nucleic Acids Res. 2011; 39:D561-D568.

33. R S, ME S, K O, et al. A travel guide to Cytoscape plugins. Nucleic Acids Res. 2012; 9:1069-1076.

34. Hanna H, Mir LM, Andre FM. In vitro osteoblastic differentiation of mesenchymal stem cells generates cell layers with distinct properties. Stem Cell Res Therapy. 2018:9:203.

35. Justesen J, Stenderup K, Ebbesen E, et al. Adipocyte tissue volume in bone marrow is increased with aging and in patients with osteoporosis. Biogerontology. 2001;2:165-71.

36. Gu Q, Tian H, Zhang K, et al. Wnt5a/FZD4 mediates the mechanical stretchinduced osteogenic differentiation of bone mesenchymal stem cells. Cell Physiol Biochem. 2018:48:215-26.

37. $H T, Y R, M T$, et al. Role of afadin in vascular endothelial growth factor- and sphingosine 1-phosphate-induced angiogenesis. Circ Res. 2010; 106:17311742.

38. F Y, Z C, H D, et al. Identification of miRNAs-genes regulatory network in diabetic nephropathy based on bioinformatics analysis. Medicine (Baltimore). 2019; 98:e16225.

39. R E, TW ON, LC H, et al. Postmenopausal osteoporosis. Medicine (Baltimore). 2016; 2:16069.

40. Wilcock A, Charlesworth S, Toller CS, et al. Denosumab: AHFS 92:24. Journal of pain and symptom management. J Pain Symptom Manage. 2018;56:295301.

41. Chapurlat R. Effects and management of denosumab discontinuation. Joint bone spine. 2018;85:515-7.

42. Lamy O, Stoll D, Aubry-Rozier B, et al. Stopping Denosumab. Curr Osteoporos Rep. 2019;17:8-15.

43. Boquete-Castro A, Gómez-Moreno G, Calvo-Guirado JL, et al. Denosumab and osteonecrosis of the jaw. A systematic analysis of events reported in clinical trials. Clinical oral implants research. 2016;27:367-75.

44. Sözen T, Özışık L. Başaran NÇJEjor An overview and management of osteoporosis. Eur J Rheumatol. 2017;4:46.

45. Miller PD. Management of severe osteoporosis. Expert Opinion on Pharmacotherapy. 2015;17:473-88.

46. LW, N N, L L, et al. H3K36 trimethylation mediated by SETD2 regulates the fate of bone marrow mesenchymal stem cells. PLoS Biol. 2018; 16:e2006522.

47. $Y F, R L, J Z$, et al. Adipogenic differentiation potential of adipose-derived mesenchymal stem cells from ovariectomized mice. Cell Prolif. 2014; 47:604614.

48. R X, X S, Y S, et al. MicroRNA-31a-5p from aging BMSCs links bone formation and resorption in the aged bone marrow microenvironment Aging Cell. 2018; 17:e12794.

49. $E$ G, A F, D R-D, et al. Paracrine mechanisms in early differentiation of human pluripotent stem cells: insights from a mathematical model. Stem Cell Res. 2018; 32:1-7. 
50. J K, A K , S C, et al. Spleen tyrosine kinase influences the early stages of multilineage differentiation of bone marrow stromal cell lines by regulating phospholipase C gamma activities. J Cell Physiol.2018; 233:2549-2559.

51. Rached MT, Kode A, Xu L, et al. FoxO1 is a positive regulator of bone formation by favoring protein synthesis and resistance to oxidative stress in osteoblasts. Cell metabolism. 2010;11:147-60.

52. Wang $Y$, Tian $\mathrm{C}$, Zheng JC. FoxO3a contributes to the reprogramming process and the differentiation of induced pluripotent stem cells. Stem Cells and Development. 2013;22:2954-63.

53. Gomez-Puerto MC, Verhagen LP, Braat AK, et al. Activation of autophagy by FOXO3 regulates redox homeostasis during osteogenic differentiation. Autophagy. 2016;12:1804-16.

54. D C, Y G, L X, et al. Bidirectional regulation of osteogenic differentiation by the FOXO subfamily of Forkhead transcription factors in mammalian MSCs. Cell Prolif. 2019; 52:e12540

55. Moriishi T, Kawai Y, Komori H, et al. Bcl2 deficiency activates FoxO through Akt inactivation and accelerates osteoblast differentiation. PLoS One. 2014;9: e86629

56. WS, W Q, B Z, et al. Overexpression of Sirt1 in mesenchymal stem cells protects against bone loss in mice by $\mathrm{FOXO} 3$ a deacetylation and oxidative stress inhibition. Metabolism. 2018; 88:61-71.

57. Attaix D, Bechet D. FoxO3 controls dangerous proteolytic liaisons. Cell metabolism. 2007:6:425-7.

58. Sanchez AM, Candau RB, Bernardi H. FoxO transcription factors: their roles in the maintenance of skeletal muscle homeostasis. Cell Mol Life Sci. 2014;71: 1657-71.

59. Hu P, Geles KG, Paik JH, et al. Codependent activators direct myoblastspecific MyoD transcription. Developmental cell. Dev Cell. 2008;15:534-46.

60. Gómez-Puerto MC, Verhagen LP, Braat AK, et al. Activation of autophagy by FOXO3 regulates redox homeostasis during osteogenic differentiation. Autophagy. 2016;12:1804-16.

61. N O, T N, I N, et al. A nucleotide variant in the promoter region of the interleukin- 6 gene associated with decreased bone mineral density. J Hum Genet. 2001; 46:267-272.

62. HW C, JS S, SE H, et al. Association of interleukin-6 promoter variant with bone mineral density in pre-menopausal women. J Hum Genet. 2003;48: 243-8.

63. Lu S, Jiao H, Xu J, et al. Downregulation of IL6 targeted MiR-376b may contribute to a positive IL6 feedback loop during early liver regeneration in mice. Cellular Physiology and Biochemistry. 2015:37:233-42.

64. R W, J R, Y S, et al. Long non-coding RNA HIF1A-AS2 facilitates adiposederived stem cells (ASCs) osteogenic differentiation through miR-665/IL6 axis via PI3KJAkt signaling pathway. Stem Cell Res Ther. 2018; 9:348.

65. Xie Z, Tang S, Ye G, et al. Interleukin-6/interleukin-6 receptor complex promotes osteogenic differentiation of bone marrow-derived mesenchymal stem cells. Stem cell research \& therapy. 2018;9:13.

66. Li M, Yan J, Chen X, et al. Spontaneous up-regulation of SIRT1 during osteogenesis contributes to stem cells' resistance to oxidative stress. J Cell Biochem. 2018;119:4928-44.

67. Yeung F, Ramirez CM, Mateos-Gomez PA, et al. Nontelomeric role for Rap1 in regulating metabolism and protecting against obesity. Cell Rep. 2013;3: 1847-56.

68. Berendsen AD, Olsen BR. How vascular endothelial growth factor-A (VEGF) regulates differentiation of mesenchymal stem cells. J Histochem Cytochem. 2014;62:103-8.

69. Zhang W, Yao C, Wei Z, et al. miR-128 promoted adipogenic differentiation and inhibited osteogenic differentiation of human mesenchymal stem cells by suppression of VEGF pathway. J Recept Signal Transduct Res. 2017;37: 217-23.

70. Xiao L, Sobue T, Esliger A, et al. Disruption of the Fgf2 gene activates the adipogenic and suppresses the osteogenic program in mesenchymal marrow stromal stem cells. Bone. 2010:47:360-70.

71. Kim S, Ahn C, Bong N, et al. Biphasic effects of FGF2 on adipogenesis. PLoS One. 2015:10:e0120073.

72. TF, Y A, R F, et al. Runx2 induces osteoblast and chondrocyte differentiation and enhances their migration by coupling with PI3K-Akt signaling. J Cell Biol. 2004; 166:85-195.

73. Y L, G C, Y H, et al. Ebselen rescues oxidative-stress-suppressed osteogenic differentiation of bone-marrow-derived mesenchymal stem cells via an antioxidant effect and the PI3K/Akt pathway. J Trace Elem Med Biol. 2019; 55:64-70.
74. X W, J C, C R, et al. GLP-1RA promotes brown adipogenesis of C3H10T1/2 mesenchymal stem cells via the PI3K-AKT-mTOR signaling pathway. Biochem Biophys Res Commun. 2018; 506:976-982.

75. $\mathrm{B} \mathrm{H}, \mathrm{M} \mathrm{L}, \mathrm{A}$ K, et al. mTOR regulates vascular smooth muscle cell differentiation from human bone marrow-derived mesenchymal progenitors. Arterioscler Thromb Vasc Biol. 2009; 29:232-238.

76. $C W, K L, J C$, et al. Osteogenesis and angiogenesis induced by porous $\beta$ CaSiO(3)/PDLGA composite scaffold via activation of AMPK/ERK1/2 and PI3K/Akt pathways. Biomaterials. 2013; 34:64-77.

\section{Publisher's Note}

Springer Nature remains neutral with regard to jurisdictional claims in published maps and institutional affiliations.
Ready to submit your research? Choose BMC and benefit from:

- fast, convenient online submission

- thorough peer review by experienced researchers in your field

- rapid publication on acceptance

- support for research data, including large and complex data types

- gold Open Access which fosters wider collaboration and increased citations

- maximum visibility for your research: over $100 \mathrm{M}$ website views per year

At $\mathrm{BMC}$, research is always in progress.

Learn more biomedcentral.com/submissions 